\title{
Double Side Signal Splitting SWIPT for Downlink CoMP Transmissions with Capacity Limited Backhaul
}

\author{
Kai Liang, Liqiang Zhao, Zhiguo Ding, and Hsiao-Hwa Chen
}

\begin{abstract}
This letter studies power allocation for simultaneous wireless information and power transfer (SWIPT) in downlink coordinated multipoint (CoMP) systems. A central unit (CU) conveys data and channel information to multiple radio remote units (RRUs) via a capacity limited backhaul. We provide a dual polarized (DP) antenna based double side signal splitting method. Specifically, signals are split up into information decoding (ID) part (UE data transmitted from CU) and energy harvesting (EH) part (deterministic data created at RRUs), which are transmitted and received via vertical and horizontal polarizations of DP antennas, respectively. Normal beamformers (such as zero forcing (ZF) and maximum ratio transmission (MRT)) are used to reduce complexity. The problem is to maximize the sum rate satisfying per-UE received power, per-backhaul-link capacity, and per-RRU power transmission constraints. Results are provided to verify the effectiveness of the proposed scheme.
\end{abstract}

Index Terms-Double side signal splitting; SWIPT; CoMP; Dual polarized antennas; Capacity limited backhaul

\section{INTRODUCTION}

$\mathbf{R}$ ADIO frequency (RF) wireless power transfer (WPT) provides controllable power supply for wireless devices. RF signals can carry both information and power, which facilitates simultaneous wireless information and power transfer (SWIPT) [1, 2]. Coordinated multipoint (CoMP) improves spectral efficiency by mitigating or exploiting multi-cell interferences. In a coordinated cluster, all radio remote units (RRUs) are connected to a central unit (CU) and transmit signals jointly to all user equipments (UEs). The CU conveys data and channel state information (CSI) to RRUs via limited capacity backhauls, compromising the performance gain of CoMP. [3] formulated a SWIPT problem for minimizing power consumption, while satisfying backhaul constraints, SINR threshold constraints, and security constraints. Dual polarized (DP) antennas provide polarization diversity and alleviate the antenna space limitation, enabling isolation between two orthogonal polarizations at RF domain for allocating more

This work was supported in part by National Natural Science Foundation of China (No. 61372070), Natural Science Basic Research Plan in Shaanxi Province of China (No. 2015JM6324), Ningbo Natural Science Foundation (2015A610117), Hong Kong, Macao and Taiwan Science \& Technology Cooperation Program of China (No. 2015DFT10160), the 111 Project (No. B08038)

Kai Liang (email: klxdu@hotmail.com) and Liqiang Zhao (email: lqzhao@mail.xidian.edu.cn) are with the State Key Laboratory of Integrated Service Networks, Xidian University, China. Zhiguo Ding (email: $z$.ding(lancaster.ac.uk) is with the School of Computing and Communications, Lancaster University, UK. Hsiao-Hwa Chen (email: hshwchen@mail.ncku.edu.tw) is with the Department of Engineering Science, National Cheng Kung University, Taiwan. antennas. To reduce complexity of beamformer design, we use zero-forcing (ZF) and maximum ratio transmission (MRT). We propose a DP antenna based double-side signal splitting power allocation method to maximize the sum rate satisfying received power, backhaul capacity, and power transmission constraints.

The contributions of this paper are summarized as follows. First, the transmission signal is split up into information decoding (ID) and energy harvesting (EH) parts. The ID part conveys UE data transmitted from CU in a hybrid CoMP model to balance backhaul loading and rate performance, while EH conveys deterministic data without consuming backhaul capacity. Second, the ID and EH parts are transmitted separately, and received via vertical and horizontal polarizations (VP and HP) of DP antennas. The EH part in this paper will not yield any interference to ID. Third, compared to traditional power splitting (PS) methods at the receiver, joint signal splitting at both sides eliminates backhaul resource consumption and interference to the $\mathrm{EH}$ part, reducing the complexity because no iterative algorithm is needed for finding PS factors. The ID part can be transmitted with a ZF beamformer to increase data rate without compromising received power, thereby further reducing the hardware complexity.

The notations are defined as follows. Lower case boldface letters represent vectors. $\mathbf{v}^{H},|\mathbf{v}|$, and $\|\mathbf{v}\|$ denote the conjugate transpose, determinant, and Euclidean norm of vector $\mathbf{v}$, respectively. $\otimes$ denotes the Kronecker product. $\mathbb{C}^{x \times y}$ and diag denote a set of complex variables of size $x \times y$ and diagonal matrix, respectively. We use " $"$ " and " $f\langle\cdot\rangle$ " denote "distributed as" and "a function of $f$ ", respectively. $\log (\cdot)$ denotes base two logarithm, and $\ln (\cdot)$ stands for the natural logarithm. We define $[x]^{+}=\max (0, x)$.

\section{SYSTEM MODEL}

Let us consider a coordinated cluster consisting of a $\mathrm{CU}$ and $J$ RRUs with each serving $K$ UEs. RRU and UE are equipped with $N_{t}>1$ and $N_{r}=1$ DP antennas, respectively. Each backhaul link has a normalized capacity of $C_{j}$ bit $/ \mathrm{s} / \mathrm{Hz}$. Assume that global CSI is perfectly known and all computations are implemented at the CU. We focus on the impact of sharing data on backhaul links and ignore the signalling overheads associated with CSI for simplicity.

We consider a flat fading channel in a time division duplex (TDD) system. In a DP multiple-input multiple-output (MIMO) channel as described in [4], the channel between 


$$
\begin{aligned}
& \mathbf{y}_{j_{k}}^{\mathcal{C}}=\sum_{i=1}^{J} \sum_{l=1}^{K} \mathbf{H}_{i, j_{k}}^{H}\left(\rho_{i_{l}}^{\mathcal{C}} \otimes \mathbf{v}_{i, i_{l}}\right) \mathbf{x}_{i_{l}}+\mathbf{z}_{j_{k}}=\sum_{i=1}^{J} \sum_{l=1}^{K}\left[\begin{array}{c}
\sqrt{p_{i_{l}}^{(v) \mathcal{C}}} \mathbf{h}_{i, j_{k}}^{(v v) H} \mathbf{v}_{i, i_{l}}^{(v)} x_{i_{l}}^{(v)}+\sqrt{p_{i_{l}}^{(h) \mathcal{C}}} \sqrt{\chi} \mathbf{h}_{i, j_{k}}^{(h v) H} \mathbf{v}_{i, i_{l}}^{(h)} x_{i_{l}}^{(h)} \\
\sqrt{p_{i_{l}}^{(h) \mathcal{C}}} \mathbf{h}_{i, j_{k}}^{(h h) H} \mathbf{v}_{i, i_{l}}^{(h)} x_{i_{l}}^{(h)}+\sqrt{p_{i_{l}}^{(v) \mathcal{C}}} \sqrt{\chi} \mathbf{h}_{i, j_{k}}^{(v h) H} \mathbf{v}_{i, i_{l}}^{(v)} x_{i_{l}}^{(v)}
\end{array}\right]+\mathbf{z}_{j_{k}} \\
& \mathbf{y}_{j_{k}}^{\mathcal{J}}=\sum_{i=1}^{J} \sum_{l=1}^{K} \mathbf{H}_{j_{k}}^{H}\left(\rho_{i_{l}}^{\mathcal{J}} \otimes \mathbf{v}_{i, i_{l}}\right) \mathbf{x}_{i_{l}}+\mathbf{z}_{j_{k}}=\sum_{i=1}^{J} \sum_{l=1}^{K}\left[\begin{array}{c}
\sqrt{p_{i_{l}}^{(v) \mathcal{J}}} \mathbf{h}_{j_{k}}^{(v v) H} \mathbf{w}_{i_{l}}^{(v)} x_{i_{l}}^{(v)}+\sqrt{p_{i_{l}}^{(h) \mathcal{J}}} \sqrt{\chi} \mathbf{h}_{j_{k}}^{(h v) H} \mathbf{w}_{i_{l}}^{(h)} x_{i_{l}}^{(h)} \\
\sqrt{p_{i_{l}}^{(h) \mathcal{J}}} \mathbf{h}_{j_{k}}^{(h h) H} \mathbf{w}_{i_{l}}^{(h)} x_{i_{l}}^{(h)}+\sqrt{p_{i_{l}}^{(v) \mathcal{J}}} \sqrt{\chi} \mathbf{h}_{j_{k}}^{(v h) H} \mathbf{w}_{i_{l}}^{(v)} x_{i_{l}}^{(v)}
\end{array}\right]+\mathbf{z}_{j_{k}} \\
& y_{j_{k}}^{(v)}=\underbrace{\sqrt{p_{j_{k}}^{(p)}} \mathbf{h}_{j, j_{k}}^{(v v) H} \mathbf{v}_{j, j_{k}}^{(v)} x_{j_{k}}^{(p)}+\sqrt{p_{j_{k}}^{(c)}} \mathbf{h}_{j_{k}}^{(v v) H} \mathbf{w}_{j_{k}}^{(v)} x_{j_{k}}^{(c)}}_{\text {ID part and mutual interference }}+\underbrace{\sum_{i=1}^{J} \sum_{l=1}^{K}\left(\sqrt{p_{i_{l}}^{(d)}} \sqrt{\chi} \mathbf{h}_{j_{k}}^{(h v) H} \mathbf{w}_{i_{l}}^{(h)} x_{i_{l}}^{(d)}\right)+z_{j_{k}}^{(v)}}_{\text {Cross polarization interference }} .
\end{aligned}
$$

$\mathrm{RRU}_{j}$ and $\mathrm{UE}_{j_{k}}$ is given by

$$
\mathbf{H}_{j, j_{k}}=\left[\begin{array}{cc}
\mathbf{h}_{j, j_{k}}^{(v v)} & \sqrt{\chi}_{\mathbf{h}_{j, j_{k}}^{(v h)}}^{(h h)} \\
\sqrt{\chi} \mathbf{h}_{j, j_{k}}^{(h v)} & \mathbf{h}_{j, j_{k}}^{(h h}
\end{array}\right]
$$

where $\chi \in[0,1]$ represents the inverse of DP antennas' crosspolar discrimination (XPD, $\in[1, \infty]$ ), which indicates the ability of antennas to distinguish two polarizations, and the superscripts $v$ and $h$ stand for VP and HP. $\mathbf{h}_{j, j_{k}}^{(a b)}$ denotes the channel between $a$-polarization of $\mathrm{RRU}_{j}$ and $b$-polarization of $\mathrm{UE}_{j, j_{k}}$, where $a, b \in\{v, h\}$.

CoMP transmission schemes can be generally classified into coordinated beamforming $(\mathrm{CB})$ and joint processing (JP). The CoMP-JP scheme achieves a higher rate but consumes more backhaul resources. In the CoMP-CB scheme, the received signal of the $k$ th UE in the $j$ th cell is given by (2), where $\mathbf{H}_{i, j_{k}} \in \mathbb{C}^{N_{t} \times 1}$ denotes a channel between RRU $i$ to UE $j_{k}$; $\mathbf{v}_{i, i_{l}}=\left[\mathbf{v}_{i, i_{l}}^{(v) H} \mathbf{v}_{i, i_{l}}^{(p) H}\right]^{H}$ is the beamformer at RRU $i$ for UE $i_{l}$ with $\left\|\mathbf{v}_{i, i_{l}}^{(v / h)}\right\|=1$; We have $\rho_{i_{l}}^{\mathcal{C}}=\operatorname{diag}\left(\sqrt{p_{i_{l}}^{(v) \mathcal{C}}}, \sqrt{p_{i_{l}}^{(h) \mathcal{C}}}\right)$, and $p_{i_{l}}^{(v / h) \mathcal{C}}$ denotes allocated powers for UE $i_{l}$ 's VP or HP, respectively; $\mathbf{x}_{i_{l}}=\left[\mathbf{x}_{i_{l}}^{(v)}, \mathbf{x}_{i_{l}}^{(h)}\right]$ denotes transmission data; $\mathbf{z}_{j_{k}}$ is complex additive white Gaussian noise (AWGN) vector with its elements obeying $z_{j_{k}}^{(v / h)} \sim \mathcal{C N}\left(0, \sigma^{2}\right)$, which includes thermal noise and signal processing noise jointly.

In CoMP-JP scheme, the received signal of $\mathrm{UE} j_{k}$ is given by (3), where $\mathbf{H}_{j_{k}}=\left[\mathbf{H}_{1, j_{k}}^{H}, \cdots, \mathbf{H}_{J, j_{k}}^{H}\right]^{H}$ is the aggregate channel; $\mathbf{w}_{j_{k}}=\left[\mathbf{w}_{j_{k}}^{(v) H} \mathbf{w}_{j_{k}}^{(g) H}\right]^{H}$ is beamformer for UE $j_{k}$ with $\mathbf{w}_{j_{k}}^{(v / h)}=\left[\mathbf{w}_{1, j_{k}}^{(v / h) H}, \cdots, \mathbf{w}_{J, j_{k}}^{(v / h) H}\right]^{H}$ and $\left\|\mathbf{w}_{j_{k}}^{(v / h)}\right\|=1$. We also have $\rho_{i_{l}}^{\mathcal{J}}=\operatorname{diag}\left(\sqrt{p_{i_{l}}^{(v) \mathcal{J}}}, \sqrt{p_{i_{l}}^{(h) \mathcal{J}}}\right)$.

\section{Double Side Signal Splitting Power ALlocATION}

Without loss of generality, we use VP and HP of DP antennas to transmit ID and EH signals, respectively. The ID part consists of UE data, which need to be transmitted from a CU through backhaul. Motivated by rate splitting [5], we further split up the ID part into private data and common data to tackle the backhaul limit issue. The EH part is deterministic data, which is created at each RRU and is known by all RRUs and UEs in the cluster, or

$$
x_{j_{k}}=[\underbrace{x_{j_{k}}^{(c)}+x_{j_{k}}^{(p)}}_{\text {ID part }}, \underbrace{x^{(d)}}_{\text {EH part }}]^{H} .
$$

The CU only conveys $x_{j_{k}}^{(p)}$ to RRU $j$ and transmits $x_{j_{k}}^{(c)}$ to all RRUs. Private and common parts are transmitted in CoMP-CB and CoMP-JP. Let us consider ZF beamformer for the ID part to reduce complexity, under an assumption of $N_{t} \geq J K$ to ensure a good interference cancellation. The EH part is transmitted in CoMP-JP with common beamformers, such as MRT. Since the EH part contains deterministic data, the corresponding cross interference can be fully eliminated during ID. This decouples the transmission of ID and EH parts.

The received signal in VP is given by (5). The cross polarization interference can be easily canceled in the ID process. However, a mutual interference between the private signal and the common signal exists. The UE splits up the private part and the common part in a serial way by successive interference cancellation (SIC). The rates of private and common data are given by

$$
\begin{aligned}
& R_{j_{k}}^{(p)}=\log \left(1+\frac{p_{j_{k}}^{(p)}\left|\mathbf{h}_{j, j_{k}}^{(v v) H} \mathbf{v}_{j, j_{k}}^{(v)}\right|^{2}}{\sigma^{2}}\right), \\
& R_{j_{k}}^{(c)}=\log \left(1+\frac{p_{j_{k}}^{(c)}\left|\mathbf{h}_{j_{k}}^{(v v) H} \mathbf{w}_{j_{k}}^{(v)}\right|^{2}}{p_{j_{k}}^{(p)}\left|\mathbf{h}_{j, j_{k}}^{(v v) H)} \mathbf{v}_{j, j_{k}}^{(v)}\right|^{2}+\sigma^{2}}\right) .
\end{aligned}
$$

The rate and received power of UE $j_{k}$ are

$$
\begin{aligned}
& R_{j_{k}}=R_{j_{k}}^{(p)}+R_{j_{k}}^{(c)} \\
& =\log \left(1+\frac{p_{j_{k}}^{(c)}\left|\mathbf{h}_{j_{k}}^{(v v) H} \mathbf{w}_{j_{k}}^{(v)}\right|^{2}+p_{j_{k}}^{(p)}\left|\mathbf{h}_{j, j_{k}}^{(v v) H} \mathbf{v}_{j, j_{k}}^{(v)}\right|^{2}}{\sigma^{2}}\right), \\
& E_{j_{k}}=\zeta \sum_{i=1}^{J} \sum_{l=1}^{K}\left(p_{i_{l}}^{(d)}\left|\mathbf{h}_{j_{k}}^{(h h) H} \mathbf{w}_{i_{l}}^{(h)}\right|^{2}\right. \\
& \left.\quad+p_{i_{l}}^{(c)} \chi\left|\mathbf{h}_{j_{k}}^{(v h) H} \mathbf{w}_{i_{l}}^{(v)}\right|^{2}+p_{i_{l}}^{(p)} \chi\left|\mathbf{h}_{i, j_{k}}^{(v h) H} \mathbf{v}_{i, i_{l}}^{(v)}\right|^{2}\right) .
\end{aligned}
$$

The backhaul capacity constraint between RRU $j$ and a CU can be expressed as

$$
B_{j}=\sum_{k=1}^{K} R_{j_{k}}^{(p)}+\sum_{j=1}^{J} \sum_{k=1}^{K} R_{j_{k}}^{(c)} \leq C_{j}
$$

The transmission power of RRU $j$ is given by

$$
P_{j}=\sum_{k=1}^{K} p_{j_{k}}^{(p)}+\sum_{i=1}^{J} \sum_{k=1}^{K}\left(p_{i_{k}}^{(c)}\left\|\mathbf{w}_{j, i_{k}}^{(v)}\right\|^{2}+p_{i_{k}}^{(d)}\left\|\mathbf{w}_{j, i_{k}}^{(h)}\right\|^{2}\right) \text {. }
$$


We formulate an optimization problem to maximize the sum rate with per-UE EH, per-backhaul-link backhaul capacity, and per-RRU transmission power constraints, or

$$
\begin{array}{cl}
\max _{p_{j_{k},}^{(p)}, p_{j_{k}}^{(c)}, p_{j_{k}}^{(d)}} & \sum_{j=1}^{J} \sum_{k=1}^{K} R_{j_{k}}, \\
\text { s.t. } & C 1: E_{j_{k}} \geq e_{j_{k}}, \\
& C 2: B_{j} \leq C_{j}, \\
& C 3: P_{j} \leq P, \\
& C 4: p_{j_{k}}^{(p)} \geq 0, p_{j_{k}}^{(c)} \geq 0, p_{j_{k}}^{(d)} \geq 0, \\
& j=1, \cdots, J, \quad k=1, \cdots, K,
\end{array}
$$

where $e_{j_{k}}$ is EH requirements for $\mathrm{UE} j_{k}$.

(12) is non-convex due to a reverse convex constraint $\mathrm{C} 2$. Note that $R_{j_{k}}^{(c)}=R_{j_{k}}-R_{j_{k}}^{(p)}$. The $B_{j}$ can be rewritten as

$$
B_{j}=\sum_{j=1}^{J} \sum_{k=1}^{K} R_{j_{k}}-\sum_{i \neq j} \sum_{k=1}^{K} R_{i_{k}}^{(p)} .
$$

Introducing a slack variable $R_{s}$, we can transform $\mathrm{C} 2$ into

$$
C 2 A: \sum_{i \neq j} \sum_{k=1}^{K} R_{i_{k}}^{(p)}+C_{j} \geq R_{s},
$$

and (12) can be reformulated as

$$
\begin{aligned}
& \max _{R_{s}, p_{j_{k}}^{(p)}, p_{j_{k}}^{(c)}, p_{j_{k}}^{(d)}} R_{s}, \\
& \text { s.t. } C 0: \sum_{j=1}^{J} \sum_{k=1}^{K} R_{j_{k}} \geq R_{s}, \\
& C 1, C 2 A, C 3, C 4, j=1, \cdots, J, k=1, \cdots, K .
\end{aligned}
$$

We claim that the two optimization problems in (12) and (15) are equivalent, because under the optimal solution $p_{j_{k}}^{(p) *}$ and $p_{j_{k}}^{(c) *}$ of (12), $\mathrm{C} 1$ is expressed as

$$
\sum_{j=1}^{J} \sum_{k=1}^{K} R_{j_{k}}\left\langle p_{j_{k}}^{(p) *}, p_{j_{k}}^{(c) *}\right\rangle=R_{s} .
$$

In this case, the solution of (12) equals to that of (15).

(15) is convex, and the Lagrangian function of (15) for any $\alpha \geq 0, \beta_{j_{k}} \geq 0, \gamma_{j} \geq 0$, and $\eta_{j} \geq 0$ is given by

$$
\begin{gathered}
\mathcal{L}=R_{s}+\alpha\left(\sum_{j=1}^{J} \sum_{k=1}^{K} R_{j_{k}}-R_{s}\right)+\sum_{j=1}^{J} \sum_{k=1}^{K} \beta_{j_{k}}\left(E_{j_{k}}-e_{j_{k}}\right) \\
+\sum_{j=1}^{J} \gamma_{j}\left[\sum_{i \neq j} \sum_{k=1}^{K}\left(R_{i_{k}}^{(p)}+C_{j}\right)-R_{s}\right]-\left[\sum_{j=1}^{J} \eta_{j}\left(P_{j}-P\right)\right] .
\end{gathered}
$$

Denote $A_{j_{k}}=\frac{1}{\sigma^{2}}\left|\mathbf{h}_{j_{k}}^{(v v) H} \mathbf{w}_{j_{k}}^{(v)}\right|^{2}$, and $A_{j, j_{k}}=$ $\frac{1}{\sigma^{2}}\left|\mathbf{h}_{j, j_{k}}^{(v v) H} \mathbf{v}_{j, j_{k}}^{(v)}\right|^{2}$. Applying the Karush-Kuhn-Tucker (KKT) optimality conditions to (17), we have

$$
p_{j_{k}}^{(p) *}=\left[\frac{A_{j_{k}} \sum_{l \neq j} \gamma_{l}}{\ln (2) Q}-\frac{1}{A_{j, j_{k}}}\right]^{+},
$$

$$
\begin{gathered}
p_{j_{k}}^{(c) *}=\left[\frac{\alpha}{\ln (2)\left(\sum_{i=1}^{J} \eta_{i}\left\|\mathbf{w}_{i, j_{k}}^{(v)}\right\|^{2}-D_{2}\right)}-\frac{p_{j_{k}}^{(p)} A_{j, j_{k}}-1}{A_{j_{k}}}\right]^{+} \\
=\frac{1}{\ln (2)}\left[\frac{\alpha}{\left(\sum_{i=1}^{J} \eta_{i}\left\|\mathbf{w}_{i, j_{k}}^{(v)}\right\|^{2}-D_{2}\right)}-\frac{A_{j, j_{k}} \sum_{l \neq j} \gamma_{l}}{Q}\right]^{+},
\end{gathered}
$$

where $Q=\left(\eta_{j}-D_{1}\right) A_{j_{k}}-A_{j, j_{k}}\left(\sum_{i=1}^{J} \eta_{i}\left\|\mathbf{w}_{i, j_{k}}^{(v)}\right\|^{2}-\right.$ $\left.D_{2}\right), D_{1}=\sum_{i=1}^{J} \sum_{l=1}^{K} \xi \chi \beta_{i_{l}}\left|\mathbf{h}_{j, i_{l}}^{(v h) H} \mathbf{v}_{j, j_{k}}^{(v)}\right|^{2}$, and $D_{2}=$ $\sum_{i=1}^{J} \sum_{l=1}^{K} \xi \chi \beta_{i_{l}}\left|\mathbf{h}_{i_{l}}^{(v h)^{H}} \mathbf{w}_{j_{k}}^{(v)}\right|^{2}$

The power allocation solution in (18) can be viewed as a multi-level water-filling problem, because the water levels of different users can be different. From (19), we can find that $p_{j_{k}}^{(c) *}$ depends on $p_{j_{k}}^{(p) *}$. Note that $\mathrm{C} 1$ must satisfy the equality; otherwise we can always reduce the power for $\mathrm{EH}$ and increase that for ID to improve the sum rate. Denote $\mathbf{p}^{(d)}=\left[p_{1_{1}}^{(d)}, \cdots, p_{J_{K}}^{(d)}\right]^{H}, \mathbf{e}=\left[e_{1_{1}}, \cdots, e_{J_{K}}\right]^{H}, \mathbf{f}_{j_{k}}=$ $\left[\left|\mathbf{h}_{j_{k}}^{H} \mathbf{u}_{1_{1}}\right|^{2}, \cdots,\left|\mathbf{h}_{j_{k}}^{H} \mathbf{u}_{J_{K}}\right|^{2}\right]$, and $\mathbf{F}=\left[f_{1_{1}}^{H}, \cdots, \mathbf{f}_{J_{K}}^{H}\right]^{H}$. The optimal $\mathbf{p}^{(d) *}$ can be obtained by solving $\mathbf{F} \mathbf{p}^{(d)}=\zeta^{-1}(\mathbf{e}-\mathbf{s})$ as

$$
\mathbf{p}^{(d) *}=\left[(\mathbf{F} \zeta)^{-1}(\mathbf{e}-\mathbf{s})\right]^{+},
$$

where $\mathbf{s}=\left[s_{1_{1}}, \cdots, s_{J_{K}}\right]^{H}$ and $s_{j_{k}}=$ $\zeta \sum_{i=1}^{J} \sum_{l=1}^{K}\left(p_{i_{l}}^{(c)} \chi\left|\mathbf{h}_{j_{k}}^{(v h) H} \mathbf{w}_{i_{l}}^{(v)}\right|^{2}+p_{i_{l}}^{(p)} \chi\left|\mathbf{h}_{i, j_{k}}^{(v h) H} \mathbf{v}_{i, i_{l}}^{(v)}\right|^{2}\right)$. The Lagrange multipliers can be updated by a gradient method as (17) is differentiable.

Note that ZF beamforming dominates the complexity of the proposed algorithm. Since ZF beamforming for each UE's common and private parts require $\mathcal{O}\left((J K-1)^{3}+\right.$ $\left.\left(J N_{t}\right)^{2}(J K-1)\right)$ and $\mathcal{O}\left((J K-1)^{3}+\left(N_{t}\right)^{2}(J K-1)\right)$ complexity, respectively, the complexity of proposed algorithm is $\mathcal{O}\left(2(J K)^{4}+\left((J+1) N_{t}\right)^{2} J K\right)$.

\section{NumericAl RESUlts}

Assume that there are two RRUs $\left(N_{t}=8\right)$ with each serving two UEs $\left(N_{r}=1\right)$. A cooperative transmission scenario for edge users is considered in this section, and thus the propagation distance from RRU $i$ to UE $j_{k}$ is $D=10+2(i-j)$ meters. Let path loss follow $20 \log _{10}(D[m])+51.4+20 \log _{10}\left(\frac{f_{c}[G H z]}{5}\right)$ [6] with the carrier frequency of $f_{c}=2.6 \mathrm{GHz}$. The propagation distance from RRU $i$ to UE $j_{k}$ is $D=10+(i-j) * 2$ meters. Let $\chi=0.1$. The RF to energy conversion efficiency is $\zeta=0.4$.

In the simulations, we considered the following existing power allocation schemes for comparison. Rate splitting [5] was used at transmitters, and power splitting and SIC were adopted at the UE with polarized signal antennas at both sides, which is denoted as RSPS. Other two methods are similar to the proposed one, but there is no rate splitting for ID part. Denote CoMP-JP transmission of ID data with DP antennas as JPTD. Denote CoMP-CB transmission of ID data with DP antennas as CBTD.

Fig. 1 shows the sum rate versus backhaul capacity under $e_{j_{k}}=3 \mu \mathrm{W}$. The rate of the proposed method outperforms that of other methods, because a hybrid CoMP model can adjust transmission mode adaptively, and the EH part yields no backhaul resource consumption and interference. For a 


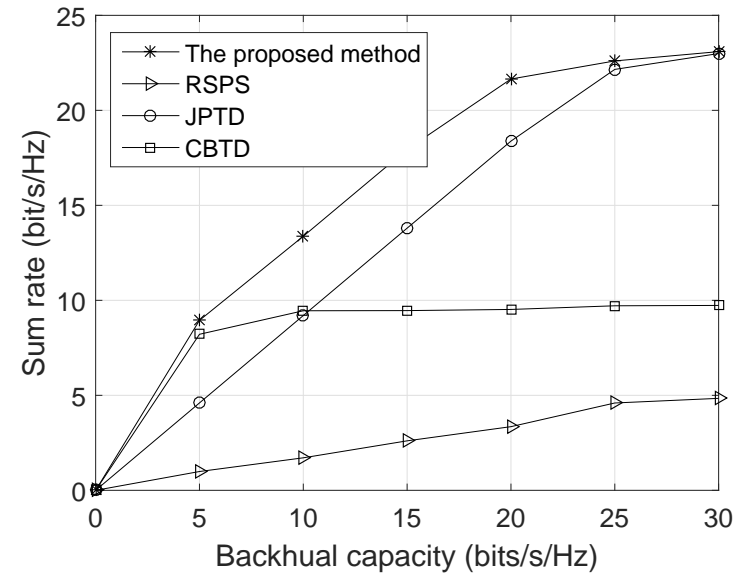

Figure 1. Sum rate performance versus backhaul capacity, where $e_{j_{k}}=$ $3 \mu \mathrm{W}$.

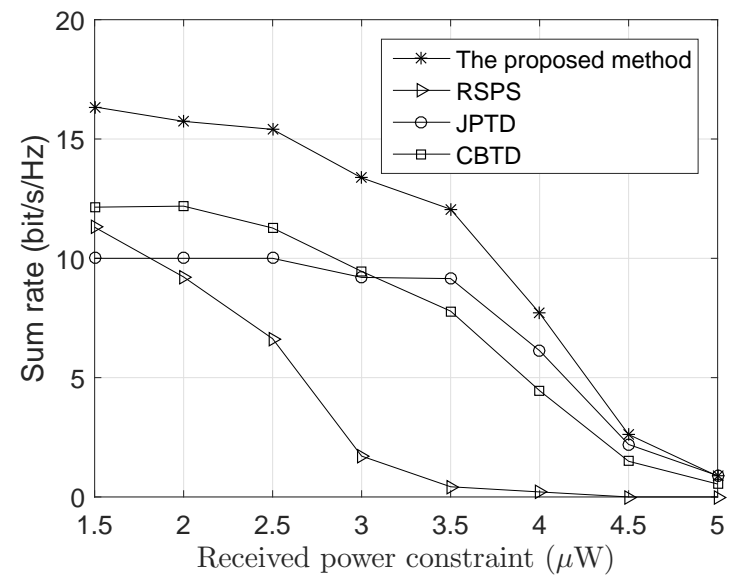

Figure 2. Sum rate performance versus received power constraint, where $C_{j}$ $=10 \mathrm{bit} / \mathrm{s} / \mathrm{Hz}$.

low backhaul capacity, CoMP-CB is the primary transmission model. CBTD achieves a similar performance as the proposed method, and better than RSPS (which splits up some power of received signal for EH). For a higher backhaul capacity, CoMP-JP is the primary transmission model, and JPTD achieves a similar rate performance as the proposed method.

Fig. 2 shows the sum rate performance versus received power constraint with capacity $C_{j}=10 \mathrm{bit} / \mathrm{s} / \mathrm{Hz}$. A higher power is allocated for EH with a larger received power constraint, and thus the sum rates of the four schemes show a downward trend with an increasing received power. The proposed method achieves the best rate performance. RSPS has the lowest rate performance because the EH part at the receiver of RSPS consumes backhaul resources.

Fig. 3 shows sum transmission power versus backhaul capacity. The power for private ID part decreases with a growing backhaul capacity, because the CoMP-CB model benefits from a lower backhaul capacity constraint. The power of common ID parts shows a reverse trend. Given the CSI, the power of EH part remains almost constant with an increasing backhaul capacity, because EH part signal dominates the received energy

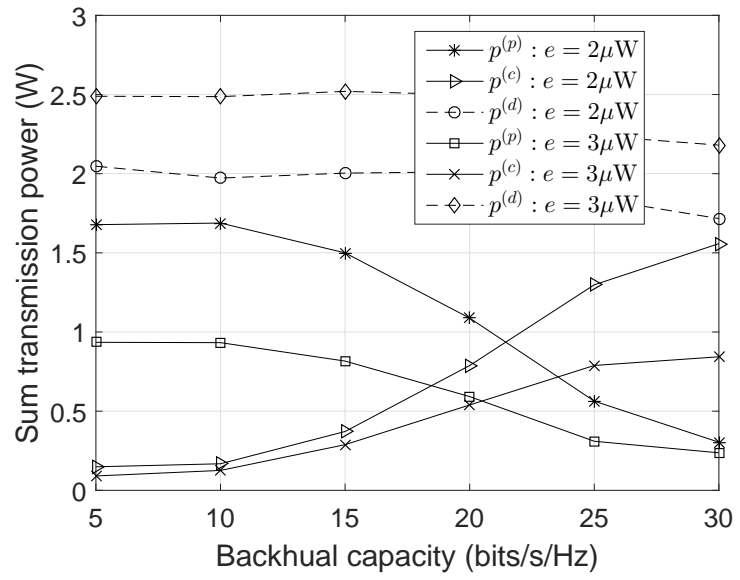

Figure 3. Sum transmission power versus backhaul capacity.

as shown in (9). The power for the ID part decreases with an increasing received power, because a higher transmission power needs to be allocated to the EH part.

\section{CONCLUSION}

In this paper, we studied a DP antenna based double side signal splitting method for SWIPT in CoMP with capacity limited backhaul, which adaptively adjusts CoMP mode, avoids backhaul cost on $\mathrm{EH}$, and reduces computational complexity. Specifically, signals are divided into ID part (using a hybrid CoMP transmission model) and EH part (consisting of deterministic data without being conveyed from the $\mathrm{CU}$ ). The ID and EH parts are transmitted separately and received through VP and HP of DP antennas, respectively. The EH parts will not yield interference for ID, which facilitates ZF beamforming for ID. Besides, the proposed method avoids additional computations caused by an exhaustive search for splitting factor as required in PS.

\section{REFERENCES}

[1] Z. Ding et al., "Application of smart antenna technologies in simultaneous wireless information and power transfer," IEEE Commun. Mag., vol. 53, pp.86-93, 2015.

[2] L. Xiao et al., "Wireless networks with RF energy harvesting: A contemporary survey," IEEE Commun. Surv. Tuts., vol. 17, no. 2, pp. 757C789, 2015.

[3] D. W. K. Ng and R. Schober, "Secure and Green SWIPT in Distributed Antenna Networks With Limited Backhaul Capacity," IEEE Trans. Wireless Commun., vol. 14, no. 9, pp. 5082-5097, 2015.

[4] T. Kim et al., "Limited Feedback Beamforming Systems for Dual-Polarized MIMO Channels," IEEE Trans. Wireless Commun., vol. 9, no. 18, pp. 3425-3439, 2010.

[5] Q. Zhang et al., "Downlink Base Station Cooperative Transmission Under Limited-Capacity Backhaul," IEEE Trans. Wireless Commun. vol. 12, no. 4, pp. 3746-3759, 2013.

[6] P. Kyösti et al., "WINNER II channel models: Part I," Inf. Soc. Technol., Munich, Germany, Tech. Rep. IST-4027756, 2008. 